Kragujevac Journal of Mathematics

Volume 41(2) (2017), Pages 239-250.

\title{
CLASSIFICATION OF TORQUED VECTOR FIELDS AND ITS APPLICATIONS TO RICCI SOLITONS
}

\author{
BANG-YEN CHEN
}

\begin{abstract}
Recently, the author defined torqued vector fields in [Kragujevac J. Math. 41(1) (2017), 93-103]. In this paper, we classify all torqued vector fields on Riemannian manifolds. Moreover, we investigate Ricci solitons with torqued potential fields. In particular, we prove that every Ricci soliton with torqued potential field is an almost quasi-Einstein manifold; and it is an Einstein manifold if and only if the potential field is a concircular vector field. Some related results on Ricci solitons are also obtained.
\end{abstract}

\section{INTRODUCTION}

A vector field $v$ on a (pseudo) Riemannian manifold $M$ is called torse-forming if it satisfies [32]

$$
\nabla_{X} v=\phi X+\psi(X) v
$$

for any vector $X \in T M$, where $\phi$ is a function, $\psi$ is a 1 -form and $\nabla$ is the Levi-Civita connection of $M$ (see also [27,30]). If the 1 -form $\psi$ in (1.1) vanishes identically, then the vector field $v$ is called concircular (cf. [4,30,31,33]). If $\phi=1$ and $\psi=0$, then the vector field $v$ is called concurrent $[30,33]$. The vector filed $v$ is called recurrent if it satisfies (1.1) with $\phi=0$. Furthermore, if $\varphi=\psi=0$, the vector field $v$ in (1.1) is called a parallel vector field.

The author made the following definition in [9].

Definition 1.1. A nowhere zero vector field $\mathcal{T}$ on a Riemannian manifold (or, more generally, in a pseudo-Riemannian manifold) is called a torqued vector field if it satisfies

Key words and phrases. Torqued vector field; twisted product; concircular vector field; Ricci soliton; almost quasi-Einstein manifold.

2010 Mathematics Subject Classification. Primary: 53C255. Secondary 53B20.

Received: September 7, 2016.

Accepted: Sepember 15, 2016. 
the following two conditions

$$
\nabla_{X} \mathcal{T}=\varphi X+\alpha(X) \mathcal{T} \text { and } \alpha(\mathcal{T})=0 .
$$

The function $\varphi$ is called the torqued function and the 1-form $\alpha$ is called the torqued form of $\mathcal{T}$.

A vector field $\xi$ on a Riemannian manifold $(M, g)$ is said to define a Ricci soliton if it satisfies

$$
\frac{1}{2} \mathcal{L}_{\xi} g+\operatorname{Ric}=\lambda g
$$

where $\mathcal{L}_{\xi} g$ is the Lie-derivative of the metric tensor $g$ with respect to $\xi$, Ric is the Ricci tensor of $(M, g)$ and $\lambda$ is a constant. We shall denote a Ricci soliton by $(M, g, \xi, \lambda)$. We call the vector field $\xi$ the potential field of the Ricci soliton. A Ricci soliton $(M, g, \xi, \lambda)$ is called shrinking, steady or expanding according to $\lambda>0, \lambda=0$, or $\lambda<0$, respectively (see, e.g., $[7,10-12,28]$ ). A trivial Ricci soliton is one for which the potential field $\xi$ is zero or Killing, in which case the metric is Einsteinian.

Compact Ricci solitons are the fixed points of the Ricci flow:

$$
\frac{\partial g(t)}{\partial t}=-2 \operatorname{Ric}(g(t))
$$

projected from the space of metrics onto its quotient modulo diffeomorphisms and scalings, and often arise as blow-up limits for the Ricci flow on compact manifolds. Further, Ricci solitons model the formation of singularities in the Ricci flow and they correspond to self-similar solutions (cf. [28]).

During the last two decades, the geometry of Ricci solitons has been the focus of attention of many mathematicians. In particular, it has become more important after Grigori Perelman applied Ricci solitons to solve the long standing Poincaré conjecture posed in 1904 (cf. [28]).

In this paper we classify all torqued vector fields on Riemannian manifolds. Moreover, we investigate Ricci solitons with torqued potential fields. In particular, we prove that if a Ricci soliton $(M, g, \mathcal{T}, \lambda)$ has torqued potential field $\mathcal{T}$, then $(M, g)$ is an almost quasi-Einstein manifold; and $(M, g)$ is an Einstein manifold if and only if the torqued potential field $\mathcal{T}$ is a concircular vector field. Some related results on Ricci solitons are also obtained.

\section{Classification of Torqued Vector Fields}

For a 1-dimensional Riemannian manifold $M$ with metric $g=d s^{2}$, every nowhere zero vector field $\mathcal{T}=\rho \partial / \partial s$ on $M$ satisfies

$$
\nabla_{X} \mathcal{T}=\frac{\partial \rho}{\partial s} X
$$

Thus every nowhere zero vector field on 1-dimensional Riemannian manifold is a torqued vector field. Therefore, throughout this article, we only consider torqued vector fields defined on Riemannian manifolds of dimension $\geq 2$. 
We recall the following definition.

Definition 2.1. The twisted product $B \times{ }_{f} F$ of two pseudo-Riemannian manifolds $\left(B, g_{B}\right)$ and $\left(F, g_{F}\right)$ is the product manifold $B \times F$ equipped with the metric

$$
g=g_{B}+f^{2} g_{F},
$$

where $f$ is a positive function on $B \times F$, which is called the twisting function.

In particular, if the function $f$ in (2.1) depends only on $B$, then it is called a warped product and the function $f$ is called the warping function.

We denote the set of lifts of vector fields on $B$ and $F$ to $B \times F$ by $\mathcal{L}(B)$ and $\mathcal{L}(F)$, respectively.

First, we recall the following result proved in [9].

Theorem 2.1. If a Riemannian manifold $M$ admits a torqued vector field $\mathcal{T}$, then $M$ is locally a twisted product $I \times_{f} F$ such that $\mathcal{T}$ is always tangent to $I$, where $I$ is an open interval.

Conversely, for each twisted product $I \times_{f} F$, there exists a torqued vector field $\mathcal{T}$ such that $\mathcal{T}$ is always tangent to $I$.

In views of Theorem 2.1 we make the following.

Definition 2.2. A torqued vector field $\mathcal{T}$ is said to be associated with a twisted product $I \times{ }_{f} F$ if $\mathcal{T}$ is always tangent to $I$.

Now, we prove the following result which classifies torqued vector fields.

Theorem 2.2. Every torqued vector field $\mathcal{T}$ associated with a twisted product $I \times{ }_{f} F$ is of the form:

$$
\mathcal{T}=\mu f \frac{\partial}{\partial s},
$$

where $s$ is an arc-length parameter of $I, \mu$ is a nonzero function on $F$, and $f$ is the twisting function.

Proof. Let $M=I \times_{f} F$ be a twisted product with twisting function $f$. Then the metric tensor of $M$ is given by

$$
g=d s^{2}+f^{2} g_{F},
$$

where $s$ is an arc-length parameter of $I$ and $g_{F}$ is the metric tensor of $F$.

Let us consider a vector field on $M$ defined by $\nu=\mu f \partial / \partial s$, where $\mu$ is a nonzero function on $F$. Then we have

$$
\begin{aligned}
& \nabla_{\mathcal{V}} \mathcal{V}=\mu \frac{\partial f}{\partial s} \mathcal{V} \\
& \nabla_{V} \mathcal{V}=(V \ln (\mu f)) \mathcal{V}+\mu \frac{\partial f}{\partial s} V .
\end{aligned}
$$


Thus $\mathcal{V}=\mu f \partial / \partial s$ is a torqued vector field satisfying (1.2) with the torqued function $\varphi=\mu \partial f / \partial s$ and the torqued form $\alpha$ satisfying

$$
\alpha(\mathcal{V})=0 \quad \text { and } \quad \alpha(V)=V(\ln \mu f)
$$

for any vector field $V \in \mathcal{L}(F)$.

Now, let $\mathcal{T}$ be an arbitrary torqued vector field associated with $I \times_{f} F$. Then, by definition, we have

$$
\nabla_{X} \mathcal{T}=\varphi X+\alpha(X) \mathcal{T} \quad \text { and } \quad \alpha(\mathcal{T})=0,
$$

Since $\mathcal{T}$ is tangent to $I$, we may put

$$
\mathcal{T}=\rho \frac{\partial}{\partial s}, \quad \rho=|\mathcal{T}|
$$

It follows (2.3) and (2.4) that

$$
\rho \varphi \frac{\partial}{\partial s}=\nabla_{\mathcal{T}} \mathcal{T}=(\mathcal{T} \rho) \frac{\partial}{\partial s}+\rho^{2} \nabla_{\frac{\partial}{\partial s}} \frac{\partial}{\partial s} .
$$

On the other hand, it follows from (2.2) that the Levi-Civita connection of $I \times_{f} F$ satisfies

$$
\nabla_{\frac{\partial}{\partial s}} \frac{\partial}{\partial s}=0, \quad \nabla_{\frac{\partial}{\partial s}} V=\nabla_{V} \frac{\partial}{\partial s}=\frac{\partial(\ln f)}{\partial s} V
$$

for any vector field $V \in \mathcal{L}(F)$ (cf. [2]). So, we derive from (2.5) and the first equation of (2.6) that

$$
\varphi=\rho \frac{\partial(\ln \rho)}{\partial s}
$$

From (2.3), (2.4) and the second equation of (2.6) we find

$$
\varphi V+\alpha(V) \mathcal{T}=\nabla_{V} \mathcal{T}=(V \rho) \frac{\partial}{\partial s}+\rho \frac{\partial(\ln f)}{\partial s} V
$$

for any vector field $V \in \mathcal{L}(F)$. Therefore we obtain

$$
\varphi=\rho \frac{\partial(\ln f)}{\partial s}, \quad \alpha(V)=V(\ln \rho) .
$$

Now, we find from (2.7) and (2.8) that

$$
\frac{\partial}{\partial s}(\ln f-\ln \rho)=0 .
$$

Hence, we get $\rho=\mu f$ for some function $\mu$ defined on $F$. Consequently, the theorem follows.

As an application of Theorem 2.2, we have the following classification of torqued vector fields on Einstein manifolds. 
Theorem 2.3. Every torqued vector field $\mathcal{T}$ on an Einstein manifold $M$ is of the form:

$$
\mathcal{T}=\zeta Z,
$$

where $Z$ is a concircular vector field on $M$ and $\zeta$ is a function satisfying $Z \zeta=0$.

Conversely, every vector field of the form (2.9) is a torqued vector field on $M$.

Proof. Let $\mathcal{T}$ be a torqued vector field on a Riemannian manifold $M$. Then Theorem 2.2 implies that $M$ is a twisted product $I \times{ }_{f} F$ such that $\mathcal{T}=\mu f \partial / \partial s$ for some nonzero function $\mu$ satisfying $\mathcal{T} \mu=0$.

In particular, if $M$ is a Einstein manifold, it is locally a warped product $I \times \tilde{f} \tilde{F}$ with warping function $\tilde{f}$, where $\tilde{F}$ is a Riemannian manifold conformally equivalent to $F$ according to [21, Corollary 1]. Therefore we obtain

$$
\mathcal{T}=\zeta \tilde{f} \frac{\partial}{\partial s},
$$

where $\zeta$ is a function satisfying $\mathcal{T} \mu=0$.

Now, if we put $Z=\tilde{f} \partial / \partial s$, then we find from (2.6) that

$$
\begin{aligned}
& \nabla_{\frac{\partial}{\partial s}} Z=\frac{\partial \tilde{f}}{\partial s} \frac{\partial}{\partial s} \\
& \nabla_{V} Z=\tilde{f} \nabla_{V} \frac{\partial}{\partial s}=\frac{\partial \tilde{f}}{\partial s} V
\end{aligned}
$$

for any vector field $V \in \mathcal{L}(F)$. Consequently, there exists a concircular vector field $Z$ such that $\mathcal{T}=\zeta Z$ with $Z \zeta=0$.

Conversely, it is easy to verify that the vector field given by (2.9) is a torqued vector field.

Another application of Theorem 2.2 is the following.

Corollary 2.1. Up to constants, there exists at most one concircular vector field associated with a warped product $I \times_{\eta} F$.

Proof. Let $Z_{1}$ and $Z_{2}$ be two concircular vector fields associated with the warped product $I \times_{\eta} F$. Then it follows from Theorem 2.2 that

$$
Z_{i}=\mu_{i} \eta \frac{\partial}{\partial s}, \quad i=1,2,
$$

for some functions $\mu_{1}, \mu_{2}$ on $F$. From (2.6), (2.10) and the definition of concircular vector field, we find

$$
\varphi_{i} V=\nabla_{V} Z_{i}=\eta\left(V \mu_{i}\right) \frac{\partial}{\partial s}+\mu_{i} \frac{\partial \eta}{\partial s} V
$$

for any vector field $V \in \mathcal{L}(F)$. Hence we obtain $V \mu_{i}=0$. Therefore the functions $\mu_{1}$ and $\mu_{2}$ are both constant. Hence $Z_{1}$ and $Z_{2}$ differ only by a constant. 
Remark 2.1. If $v_{1}$ and $v_{2}$ are two concurrent vector fields on a Riemannian manifold $M$, then we have $\nabla_{X} v_{i}=X$ for $i=1,2$. Hence

$$
\nabla_{X}\left(v_{1}-v_{2}\right)=0
$$

Thus we get $v_{2}=v_{1}+c$, where $c$ is a parallel vector (i.e., $\nabla c=0$ ). Consequently, up to parallel vectors, there exists at most one concurrent vector field on a Riemannian manifold.

\section{Applications to Ricci Solitons}

A pseudo Riemannian manifold $(M, g)$ is called a quasi-Einstein manifold if its Ricci tensor Ric satisfies

$$
\text { Ric }=a g+b \alpha \otimes \alpha,
$$

for functions $a, b$, and 1-form $\alpha$.

It is well-known that quasi-Einstein manifolds arose during the study of exact solutions of the Einstein field equations as well as during considerations of quasiumbilical hypersurfaces of conformally flat spaces. In particular, it was well-known that Robertson-Walker spacetimes are quasi-Einstein (see, e.g., [17]).

For results on quasi-Einstein hypersurfaces in spaces of constant curvature we refer to $[16,18,19]$ amongst others. More recently, quasi-Einstein manifolds were investigated amongst others in [13,20,23-25].

Remark 3.1. As far as I know, the term "quasi-Einstein" was already used in 1980 (see $[22,29])$.

A pseudo Riemannian manifold $(M, g)$ is called a generalized quasi-Einstein [15] (resp., mixed quasi-Einstein [26] or nearly quasi-Einstein [14]) manifold if its Ricci tensor satisfies

$$
\begin{aligned}
\text { Ric } & =a g+b \alpha \otimes \alpha+c \beta \otimes \beta, \\
(\text { resp. }, & \text { Ric }=a g+b \alpha \otimes \beta+c \beta \otimes \alpha \text { or } \operatorname{Ric}=a g+b E)
\end{aligned}
$$

where $a, b, c$ are functions, $\alpha, \beta$ are 1 -forms, and $E$ is a non-vanishing symmetric $(0,2)$-tensor on $M$.

In this paper, we make the following.

Definition 3.1. A pseudo Riemannian manifold is called almost quasi-Einstein if its Ricci tensor satisfies

$$
\operatorname{Ric}=a g+b(\beta \otimes \gamma+\gamma \otimes \beta)
$$

for some functions $a, b$ and 1-forms $\beta$ and $\gamma$.

In this section, we study Ricci solitons with torqued potential field.

Proposition 3.1. If the potential field of a Ricci soliton $(M, g, \mathcal{T}, \lambda)$ is a torqued vector field $\mathcal{T}$, then $(M, g)$ is an almost quasi-Einstein manifold. 
Proof. Assume that $(M, g, \mathcal{T}, \lambda)$ is a Ricci soliton whose potential field is a torqued vector field. Then (1.2) holds, which implies

$$
\begin{aligned}
\left(\mathcal{L}_{\mathcal{T}} g\right)(X, Y) & =g\left(\nabla_{X} \mathcal{T}, Y\right)+g\left(\nabla_{Y} \mathcal{T}, X\right) \\
& =2 \varphi g(X, Y)+\alpha(X) g(\mathcal{T}, Y)+\alpha(Y) g(\mathcal{T}, X)
\end{aligned}
$$

for any vector fields $X, Y$ tangent to $M$.

By combining (1.3) and (3.1), we find

$$
\operatorname{Ric}(X, Y)=(\lambda-\varphi) g(X, Y)-\frac{1}{2} \alpha(X) g(\mathcal{T}, Y)-\frac{1}{2} \alpha(Y) g(\mathcal{T}, X) .
$$

If we denote the dual 1 -form of $\mathcal{T}$ by $\gamma$, then (3.2) yields

$$
\text { Ric }=(\lambda-\varphi) g-\frac{1}{2}(\alpha \otimes \gamma+\gamma \otimes \alpha) .
$$

Therefore $(M, g)$ is an almost quasi-Einstein manifold.

An immediate consequence of (3.1) is the following.

Corollary 3.1. A torqued vector field $\mathcal{T}$ on a Riemannian manifold $M$ is a Killing vector field if and only if $\mathcal{T}$ is a recurrent vector field satisfies

$$
\nabla_{X} \mathcal{T}=\alpha(X) \mathcal{T} \quad \text { and } \quad \alpha(\mathcal{T})=0,
$$

where $\alpha$ is a 1-form.

Recall that a Ricci soliton $(M, g, \xi, \lambda)$ with $\operatorname{dim} M \geq 3$ is called trivial if $(M, g)$ is an Einstein manifold.

The following result provides a very simple characterization for a Ricci soliton with torqued potential field to be trivial.

Theorem 3.1. A Ricci soliton $(M, g, \mathcal{T}, \lambda)$ with torqued potential field $\mathcal{T}$ is trivial if and only if $\mathcal{T}$ is a concircular vector field.

Proof. Assume that $(M, g, \mathcal{T}, \lambda)$ is a Ricci soliton on a Riemannian $n$-manifold with a torqued potential field $\mathcal{T}$. Then (3.2) holds for any vector fields $X, Y$ tangent to $M$. In particular, we have

$$
\operatorname{Ric}(\mathcal{T}, V)=-\frac{1}{2} \alpha(\mathcal{T}) g(\mathcal{T}, V)-\frac{1}{2} \alpha(V) g(\mathcal{T}, \mathcal{T})
$$

for any vertical vector field $V$. Since $\mathcal{T}$ is torqued, we have $\alpha(\mathcal{T})=0$. Thus

$$
\operatorname{Ric}(\mathcal{T}, V)=-\frac{1}{2} \alpha(V) g(\mathcal{T}, \mathcal{T}),
$$

for any vector field $V$ perpendicular to $\mathcal{T}$. Thus, if $(M, g)$ is an Einstein manifold, then (3.3) gives $\alpha(V) g(\mathcal{T}, \mathcal{T})=0$. Since $\mathcal{T}$ is nowhere zero, we obtain $\alpha(V)=0$ for any vector $V$ orthogonal to $\mathcal{T}$. Combining this with $\alpha(\mathcal{T})=0$ gives $\alpha=0$. Hence the potential field $\mathcal{T}$ is a concircular vector field. 
Conversely, if $(M, g, Z, \lambda)$ is a Ricci soliton with concircular vector field $Z$, then we have $\nabla_{X} Z=\varphi X$ for some function $\varphi$. Thus

$$
\left(\mathcal{L}_{Z} g\right)(X, Y)=g\left(\nabla_{X} Z, Y\right)+g\left(\nabla_{Y} Z, X\right)=2 \varphi g(X, Y)
$$

for any $X, Y$ tangent to $M$. After combining this with (1.3) we find

$$
\operatorname{Ric}(X, Y)=(\lambda-\varphi) g(X, Y)
$$

which implies that $(M, g)$ is an Einstein manifold. Consequently, the Ricci soliton is a trivial one.

Remark 3.2. Let $(M, g, \xi, \lambda)$ be a Ricci soliton with $\operatorname{dim} M \geq 3$. If the potential field $\xi$ of the Ricci soliton is a concircular vector field $Z$, then $Z$ must satisfies $\nabla_{X} Z=c X$ for some constant $c$. This can be seen as follows: Since $\lambda$ given in (3.4) is a constant and $\operatorname{dim} M \geq 3$, the function $\varphi$ in (3.4) is also a constant since $(M, g)$ is an Einstein manifold.

\section{Ricci Solitons with Canonical Torqued Potential Field}

In view of Theorem 2.1 we give the following.

Definition 4.1. For a twisted product $I \times_{f} F$, the torqued vector field $f \partial / \partial s$ is called the canonical torqued vector field of $I \times{ }_{f} F$, where $s$ is an arc-length parameter on $I$. We denote the canonical vector field $f \partial / \partial s$ by $\mathcal{T}_{c a}^{f}$.

Recall from Theorem 2.1 that if a Riemannian manifold $M$ admits a torqued vector field, then $M$ is locally a twisted product $I \times{ }_{f} F$, where $f$ is the twisting function and $F$ is a Riemannian $(n-1)$-manifold.

Now, we prove the following.

Theorem 4.1. If $\left(I \times_{f} F, g, \mathcal{T}_{c a}^{f}, \lambda\right)$ is a Ricci soliton with the canonical torqued vector field $\mathcal{T}_{c a}^{f}$ as its potential field, then we have:

(a) $\mathcal{T}_{c a}^{f}$ is a concircular vector field and

(b) $\left(I \times_{f} F, g\right)$ is an Einstein manifold.

Proof. Assume that $\left(I \times_{f} F, g, \mathcal{T}_{c a}^{f}, \lambda\right)$ is a Ricci soliton with the canonical torqued vector field $\mathcal{T}_{c a}^{f}$ as its potential field. Then (1.2) holds for $\mathcal{T}=\mathcal{T}_{c a}^{f}$, which implies

$$
\left(\mathcal{L}_{\mathcal{T}_{c a}^{f}} g\right)(X, Y)=2 \varphi g(X, Y)+\alpha(X) g\left(\mathcal{T}_{c a}^{f}, Y\right)+\alpha(Y) g\left(\mathcal{T}_{c a}^{f}, X\right),
$$

for any $X, Y$ tangent to $M$. Combining (1.3) and (4.1) gives

$$
\operatorname{Ric}(X, Y)=(\lambda-\varphi) g(X, Y)-\frac{1}{2} \alpha(X) g\left(\mathcal{T}_{c a}^{f}, Y\right)-\frac{1}{2} \alpha(Y) g\left(\mathcal{T}_{c a}^{f}, X\right) .
$$

Since $\mathcal{T}_{c a}^{f}$ is the canonical torqued vector field on $I \times_{f} F$, we have

$$
g=d s^{2}+f^{2} g_{F}, \quad \mathcal{T}_{c a}^{f}=f \frac{\partial}{\partial s} .
$$


Hence it follows from (2.8) that

$$
\varphi=f_{s}, \quad \alpha(V)=V(\ln f),
$$

for any vector field $V \in \mathcal{L}(F)$. Because $\alpha\left(\mathcal{T}_{\text {ca }}^{f}\right)=0$, we derive from (4.2), (4.3) and (4.4) that

$$
\begin{aligned}
\operatorname{Ric}\left(\mathcal{T}_{c a}^{f}, \mathcal{T}_{c a}^{f}\right) & =\left(\lambda-f_{s}\right) f^{2}, \\
\operatorname{Ric}\left(\mathcal{T}_{c a}^{f}, V\right) & =-\frac{V\left(f^{2}\right)}{4}, \\
\operatorname{Ric}(V, W) & =\left(\lambda-f_{s}\right) g(V, W),
\end{aligned}
$$

for vector fields $V, W \in \mathcal{L}(F)$. Since $M$ is an open portion of the twisted product $I \times{ }_{f} F$, we also have (cf. e.g., [21])

$$
\begin{aligned}
\operatorname{Ric}\left(\mathcal{T}_{c a}^{f}, \mathcal{T}_{c a}^{f}\right)= & (1-n) f f_{s s}, \quad n=\operatorname{dim} M, \\
\operatorname{Ric}\left(\mathcal{T}_{c a}^{f}, V\right)= & (2-n) V\left(f_{s}\right), \\
\operatorname{Ric}(V, W)= & \operatorname{Ric}^{F}(V, W)+(3-n)\left\{V W(\ln f)-\left(\nabla_{V}^{F} W\right)(\ln f)\right\} \\
& +(n-1) V(\ln f) W(\ln f)-g(V, W)\{\Delta(\ln f) \\
& +g(\nabla(\ln f), \nabla(\ln f))\},
\end{aligned}
$$

for any vector fields $V, W \in \mathcal{L}(F)$, where $\Delta(\ln f)$ is the Laplacian of $\ln f$ on $M$.

From (4.5) and (4.7) we find

$$
(1-n) f_{s s}=\left(\lambda-f_{s}\right) f \text {. }
$$

On the other hand, it follows from (4.6) and (4.8) that

$$
4(n-2) V\left(f_{s}\right)=V\left(f^{2}\right),
$$

for any vector field $V \in \mathcal{L}(F)$.

After solving the differential equation (4.9) we get

$$
4(n-2) f_{s}-f^{2}=k(s),
$$

for some function $k=k(s)$. Now, by taking the derivative of (4.11) we obtain

$$
f_{s s}=\frac{2 f f_{s}+k^{\prime}(s)}{4(n-2)} .
$$

After substituting (4.12) into (4.10) we find

$$
(n-3) f_{s}=(n-1) \frac{k^{\prime}(s)}{2 f}+2(n-2) \lambda .
$$

By solving (4.11) for $f_{s}$ and by substituting it into (4.13) we obtain

$$
(n-3) f^{3}-\left[(n-3) k(s)+8(n-2)^{2} \lambda\right] f=2\left(n^{2}-3 n+2\right) k^{\prime}(s) .
$$

Equation (4.14) implies that the twisting function $f$ is a function depending only on $s$, not on $F$. Hence $I \times{ }_{f} F$ is a warped product. Therefore, the canonical torqued 
vector field $\mathcal{T}_{c a}^{f}$ is a concircular vector field. Consequently, by Theorem 3.1, $\left(I \times_{f} F, g\right)$ is an Einstein manifold.

Remark 4.1. Ricci solitons $(M, g, Z, \lambda)$ with concircular potential field $Z$ have been completely determined in [5, Theorem 5.1].

Remark 4.2. If the potential field of the Ricci soliton defined on $\left(I \times_{f} F, g\right)$ in Theorem 4.1 is an arbitrary torqued vector field $\mathcal{T}$ associated with $I \times_{f} F$, then it follows from Theorem 2.2 that $\mathcal{T}=\mu f \partial / \partial s$ for some function $\mu$ defined on $F$. In this case, we may consider the twisted product $I \times \tilde{f} \tilde{F}$ instead, where $\tilde{f}=\mu f$ and $\tilde{F}$ is the manifold $F$ with metric $\tilde{g}_{F}=\mu^{-2} g_{F}$. Then $(I \times \tilde{f} \tilde{F}, \tilde{g}, \mathcal{T}, \lambda)$ with $\tilde{g}=d s^{2}+\tilde{f}^{2} \tilde{g}_{F}$ is a Ricci soliton whose potential field $\mathcal{T}$ is the canonical torqued vector field $\mathcal{T}_{c a}^{\tilde{f}}$ of $I \times_{\tilde{f}} \tilde{F}$.

An application of Theorem 4.1 is the following.

Corollary 4.1. Let $\left(I \times_{f} F, g, \mathcal{T}_{c a}^{f}, \lambda\right)$ be a steady Ricci solitons with the canonical torqued vector field $\mathcal{T}_{\text {ca }}^{f}$ as its potential field. If $\operatorname{dim} F \geq 2$, then we have:

(a) $\mathcal{T}_{c a}^{f}$ is a parallel vector field,

(b) $f$ is a constant, say $c$,

(c) $\left(I \times_{c} F, g\right)$ is a Ricci-flat manifold, and

(d) $F$ is also Ricci-flat.

Proof. Let $\left(I \times_{f} F, g, \mathcal{T}_{c a}^{f}, \lambda\right)$ be a Ricci soliton with the canonical torqued vector field $\mathcal{T}_{c a}^{f}$ as its potential field. Then it follows from Theorem 4.1 that $\mathcal{T}_{c a}^{f}$ is a concircular vector field. Thus we have

$$
\nabla_{X} \mathcal{T}_{c a}^{f}=\varphi X
$$

for some function $\varphi$. Thus we find from (4.2) that the Ricci tensor of $I \times_{f} F$ satisfies

$$
\operatorname{Ric}(X, Y)=(\lambda-\varphi) g(X, Y) \text {, }
$$

for any vector fields $X, Y$. Since $\operatorname{dim} F \geq 2$ and $I \times_{f} F$ is an Einstein manifold, (4.16) implies that $\varphi$ is a constant, say $b$. Therefore, it follows from (4.15) that

$$
\nabla_{X} \mathcal{T}_{c a}^{f}=b X
$$

Now, suppose that the Ricci soliton is steady. Then we have $\lambda=0$. Hence Eq. (4.16) yields

$$
\operatorname{Ric}(X, Y)=-b g(X, Y)
$$

for any vector fields $X, Y$.

On the other hand, it follows from (4.15) that the Riemann curvature tensor $R$ satisfies

$$
R\left(X, \mathcal{T}_{c a}^{f}\right) \mathcal{T}_{c a}^{f}=\nabla_{X} \nabla_{\mathcal{T}_{c a}^{f}} \mathcal{T}_{c a}^{f}-\nabla_{\mathcal{T}_{c a}^{f}} \nabla_{X} \mathcal{T}_{c a}^{f}-\nabla_{\left[X, \mathcal{T}_{c a}^{f}\right]} \mathcal{T}_{c a}^{f}=0
$$

Thus $\operatorname{Ric}\left(\mathcal{T}_{c a}^{f}, \mathcal{T}_{c a}^{f}\right)=0$. Therefore we get $b=0$ and hence $I \times_{f} F$ is a Ricci-flat manifold by (4.18). Moreover, it follows from (4.17) that $\mathcal{T}_{c a}^{f}$ is a parallel vector field. Moreover, we conclude from (4.5) that $f_{s}=0$. Thus $f$ is a constant. Therefore $F$ is Ricci-flat as well. 


\section{REFERENCES}

[1] B.-Y. Chen, Geometry of Submanifolds, Marcel Dekker, New York, 1973.

[2] B.-Y. Chen, Geometry of Submanifolds and its Applications, Science University of Tokyo, Tokyo, 1981.

[3] B.-Y. Chen, Pseudo-Riemannian Geometry, $\delta$-Invariants and Applications, World Scientific, New Jersey, 2011.

[4] B.-Y. Chen, A simple characterization of generalized Robertson-Walker spacetimes, Gen. Relativity Gravitation 46(12) (2014), Article ID 1833.

[5] B.-Y. Chen, Some results on concircular vector fields and their applications to Ricci solitons, Bull. Korean Math. Soc. 52 (2015), 1535-1547.

[6] B.-Y. Chen, Differential geometry of rectifying submanifolds, Int. Electron. J. Geom. 9(2) (2016), $1-8$.

[7] B.-Y. Chen, A survey on Ricci solitons on Riemannian submanifolds, Contemp. Math. 674 (2016), 27-39.

[8] B.-Y. Chen, Topics in differential geometry associated with position vector fields on Euclidean submanifolds, Arab J. Math. Sci. (2017), (in press).

[9] B.-Y. Chen, Rectifying submanifolds of Riemannian manifolds and torqued vector fields, Kragujevac J. Math. 41(1) (2017), 93-103.

[10] B.-Y. Chen and S. Deshmukh, Geometry of compact shrinking Ricci solitons, Balkan J. Geom. Appl. 19(1) (2014), 13-21.

[11] B.-Y. Chen and S. Deshmukh, Classification of Ricci solitons on Euclidean hypersurfaces, Internat. J. Math. 25(11) (2014), Article ID 1450104.

[12] B.-Y. Chen and S. Deshmukh, Ricci solitons and concurrent vector fields, Balkan J. Geom. Appl. 20(1) (2015), 14-25.

[13] M. C. Chaki and R. K. Maity, On quasi Einstein manifold, Publ. Math. Debrecen 57 (2000), 297-306.

[14] U. C. De and A. K. Gazi, On nearly quasi Einstein manifolds, Novi Sad J. Math. 38(2) (2008), $115-121$.

[15] U. C. De and G. C. Ghosh, On generalized quasi-Einstein manifolds, Kyungpook Math. J. 44 (2004), 607-615.

[16] R. Deszcz, M. Glogowska, M. Hotloś and Z. Sentürk, On certain quasi-Einstein semisymmetric hypersurfaces, Ann. Univ. Sci. Budapest. Eötvös Sect. Math. 41 (1998), 151-164.

[17] R. Deszcz, M. Glogowska, J. Jelowicki, M. Petrović-Torgašev and G. Zafindratafa, On Riemann and Weyl compatible tensors, Publ. Inst. Math. (Beograd) (N.S.) 94(108) (2013), 111-124.

[18] R. Deszcz, M. Hotloś, and Z. Sentürk, On curvature properties of quasi-Einstein hypersurfaces in semi-Euclidean spaces, Soochow J. Math. 27 (2001), 375-389.

[19] R. Deszcz and L. Verstraelen, Hypersurfaces of semi-Riemannian conformally flat manifolds, in: Geometry and Topology of Submanifolds, III, World Sci., River Edge, NJ, 1991, 131-147.

[20] D. Dumitru, On quasi-Einstein warped products, Jordan J. Math. Stat. 5 (2012), 85-95.

[21] M. Fernández-López, E. Garcia-Rio, D. N. Kupeli and B. Ünal, A curvature condition for a twisted product to be a warped product, Manuscripta Math. 106(2) (2001), 213-217.

[22] S. I. Goldberg and I. Vaisman, On compact locally conformal Kaehler manifolds with nonnegative sectional curvature, Ann. Fac. Sci. Toulouse Math. Ser. V, 2(2) (1980), 117-123.

[23] S. K. Jana, A. A. Shaikh, On quasi-Einstein spacetime with space-matter tensor, Lobachevskii J. Math. 33 (2012), 255-261.

[24] V. A. Kiosak, On the conformal mappings of quasi-Einstein spaces, J. Math. Sci. 184 (2012), $12-18$.

[25] R. S. Lemence, On sectional curvature of quasi-Einstein manifolds, Int. J. Math. Analysis 6 (2012), 1105-1113. 
[26] S. Mallick and U. C. De, On mixed quasi-Einstein manifolds, Ann. Univ. Sci. Budapest. Eötvös Sect. Math. 57 (2014), 59-73.

[27] A. Mihai and I. Mihai, Torse forming vector fields and exterior concurrent vector fields on Riemannian manifolds and applications, J. Geom. Phys. 73 (2013), 200-208.

[28] J. Morgan and G. Tian, Ricci Flow and the Poincaré Conjecture, Clay Mathematics Monographs, 5, Cambridge, MA, 2014.

[29] V. M. Nikolaenko, Higgs mesons in gauge gravitation, Theoret. and Math. Phys. 42(2) (1980), 128-133.

[30] J. A. Schouten, Ricci Calculus, Springer-Verlag, Berlin, 1954.

[31] K. Yano, Concircular geometry. I, Concircular transformations, Proc. Imp. Acad. Tokyo 16 (1940), 195-200.

[32] K. Yano, On torse forming direction in a Riemannian space, Proc. Imp. Acad. Tokyo 20 (1944), 340-345.

[33] K. Yano and B.-Y. Chen, On the concurrent vector fields of immersed manifolds, Kodai Math. Sem. Rep. 23 (1971), 343-350.

Department of Mathematics

Michigan State University

619 RED CEDAR ROAD

EAst LAnsing, Michigan 48824-1027, U.S.A.

E-mail address: bychen@math.msu.edu 